RESEÑA DE LIBRO

\title{
PLANEAMIENTO ESTRATÉGICO PROSPECTIVO: MÉTODOS MACTOR Y SMIC
}

PROSPECTIVE STRATEGIC PLANNING: MACTOR AND SMIC METHODS

PLANEJAMENTO ESTRATÉGICO PROSPECTIVO: MÉTODOS MACTOR E SMIC

Cristian Germán Hernándezii

\section{Forma de citar}

Hernández, Cristian G. (2019) Planeamiento estratégico prospectivo: métodos MACTOR y SMIC. Dimensión Empresarial, 18(1). DOI: 10.15665/dem.v18i(1).2127

\section{Resumen}

Los autores, Quinteros \& Hamann (2017) presentan algunos temas sobre planeación y prospectiva estratégica a lo largo del texto. A lo largo de sus capítulos se presenta un marco referencial, el instructivo de aplicación de los métodos MACTOR y SMIC, el modelamiento de macroentornos y de organizaciones individualizadas, el proceso de análisis prospectivo en espectros sociales u organizacionales y la implementación del análisis prospectivo como plan de negocios de una empresa. Igualmente se adjunta un anexo con formatos para elaborar herramientas de recolección de datos que faciliten la consecución de un estudio fundamentado en la escuela francesa de prospectiva. Palabras clave: planeación, prospectiva, administración, MACTOR, SMIC.

\section{Abstract}

The authors, Quinteros \& Hamann (2017) present some topics on strategic planning and prospective throughout the text. Throughout its chapters a referential framework is presented, the instructions for applying the MACTOR and SMIC methods, the modeling of macro environments and individualized organizations, the process of prospective analysis in social or organizational spectra and the implementation of prospective analysis as a plan of business of a company. Also attached is an annex with formats to develop data collection tools that facilitate the achievement of a study based on the French prospective school. Keywords: planning, prospective, administration, MACTOR, SMIC.

\section{Resumo}

Os autores, Quinteros \& Hamann (2017) apresentam alguns tópicos sobre planejamento estratégico e prospectivo ao longo do texto. Ao longo de seus capítulos, é apresentada uma estrutura referencial, as instruções para a aplicação dos métodos MACTOR e SMIC, a modelagem de ambientes macro e organizações individualizadas, o processo de análise prospectiva em espectros sociais ou organizacionais e a implementação da análise prospectiva como plano. de negócios de uma empresa. Também é anexado um anexo com formatos para desenvolver ferramentas de coleta de dados que facilitam a realização de um estudo baseado na escola prospectiva francesa. Palavras-chave: planejamento, prospectivo, administração, MACTOR, SMIC. 


\section{PLANEAMIENTO ESTRATÉGICO PROSPECTIVO: MÉTODOS MACTOR Y SMIC}

La lectura de Quinteros \& Hamann (2017) muestra la vinculación entre su formación académica, su experiencia profesional y el tema del texto. Esto se puede evidenciar al revisar la tabla de contenidos, la cual fue estructurada bajo una premisa pedagógica que favorece la comprensión del orden sistémico de la planeación con enfoque prospectivo. Para este fin, se plantea un marco referencial considerable, así como explicaciones por fases del uso de software (LIPSOR) y la aplicación de métodos prospectivos. De forma adicional, se presentan casos desarrollados en espectros sociales y empresariales y una compilación de formatos de encuesta para la consulta a expertos.

La estructura del libro comprende cinco apartados. En ellos se encuentra el marco referencial que esclarece los conceptos involucrados en este tipo de planeación estratégica organizacional, ligada a los estudios de futuro, específicamente a los métodos de análisis de la escuela francesa. También se estudian las herramientas de prospectiva y las matrices empresariales que aportan la información necesaria para el adecuado desarrollo de dos componentes fundamentales del plan estratégico: el diagnóstico y la propuesta de implementación.

Las matrices empresariales se encuentran explicadas textualmente con el apoyo de citas de los autores más relevantes en el ámbito $y$, conjuntamente, se proporcionan formatos para su desarrollo y ejemplos de casos. A continuación se presenta una tabla resumen:

En la elaboración de un plan estratégico, la consecución de la visión es lo primordial. Por lo tanto, el análisis de la competitividad de la organización y la generación de valor son aspectos fundamentales en el proceso. Porter

Table 1. Matrices empresariales para la planeación estratégica prospectiva

\begin{tabular}{|l|l|}
\hline Nombre de la matriz & Descripción \\
\hline Matriz IE & $\begin{array}{l}\text { Se compone por el análisis interno y externo. Para esto se estudia los factores internos } \\
\text { determinantes de éxito: fortalezas y debilidades; así como los factores externos } \\
\text { determinantes de éxito: oportunidades y amenazas. Las valoraciones se realizan con el } \\
\text { apoyo de las matrices EFI y EFE. }\end{array}$ \\
\hline $\begin{array}{l}\text { Matriz McKinsey o de Cartera } \\
\text { Multifactor }\end{array}$ & $\begin{array}{l}\text { Se basa en dos factores: la atracción del mercado y la posición competitiva de la } \\
\text { empresa. Se plantea que esta tendrá éxito a medida que penetre en mercados atractivos } \\
\text { y tenga la mezcla de atributos competitivos para sobresalir. }\end{array}$ \\
\hline $\begin{array}{l}\text { Matriz Boston Consulting Group } \\
\text { (BCG) }\end{array}$ & $\begin{array}{l}\text { Se estructura ubicando cada unidad productiva de negocio con la finalidad de } \\
\text { evaluarlas con base en el índice de crecimiento del mercado y la participación } \\
\text { relativa de la empresa. }\end{array}$ \\
\hline y Evaluación de la Acción (PEYEA) & $\begin{array}{l}\text { Los ejes de esta matriz contemplan dos dimensiones internas: fuerza financiera y } \\
\text { ventaja competitiva, y dos dimensiones externas: fuerza de la industria y estabilidad } \\
\text { del ambiente. Posterior a su elaboración, se puede optar por adoptar una estrategia } \\
\text { activa, conservadora, defensiva o competitiva. }\end{array}$ \\
\hline Matriz de la Gran Estrategia & $\begin{array}{l}\text { Consiste en una compilación de estrategias genéricas que se pueden emplear } \\
\text { dependiendo de la velocidad con la cual crece el mercado y el nivel de } \\
\text { fortaleza con respecto a la posición competitiva de la empresa. }\end{array}$ \\
\hline
\end{tabular}




\begin{tabular}{|l|l|}
\hline Nombre de la matriz & Descripción \\
\hline Matriz FODA & $\begin{array}{l}\text { Consiste en la identificación de fortalezas, oportunidades, debilidades y amenazas, } \\
\text { y su elaboración se cataloga como un método cualitativo de planeación. Ayuda a } \\
\text { desarrollar cuatro tipos de estrategias que derivan del cruce de los cuatro aspectos } \\
\text { que se analizan. }\end{array}$ \\
\hline $\begin{array}{l}\text { Matriz Cuantitativa de la } \\
\text { Planificación Estratégica (MCPE) }\end{array}$ & $\begin{array}{l}\text { Es una herramienta con mayor valor objetivo, puesto que implica } \\
\text { ponderaciones en las estrategias seleccionadas; es decir, se realiza una } \\
\text { evaluación con base en las posibilidades de la organización, sus prioridades y } \\
\text { factores clave. }\end{array}$ \\
\hline
\end{tabular}

Fuente: Elaboración del autor con base en el texto de Quinteros \& Hamann (2017).

(2010) lo planteó como la herramienta promotora de ventaja competitiva denominada como Cadena de Valor, la cual se explica en el texto mediante estructuras específicas y genéricas de OSIPTEL (Organismo Superior de Inversión Privada en Telecomunicaciones). De igual manera, se hace mención al BSC (Balanced Scorecard), diseñado por Kaplan \& Norton (1996), entendido como un instrumento de control estratégico que surge de la visión, misión yestrategias determinadas por la empresa. EI BSC contempla cuatro perspectivas: finanzas, cliente, procesos internos e innovación y aprendizaje.

El primer acercamiento que hacen los autores a los métodos prospectivos se realiza mediante la explicación del análisis estructural MICMAC (Matriz de Impactos Cruzados y Multiplicación Aplicada a una Clasificación). En este punto, se busca diagnosticar la relación que existe entre las variables involucradas en el estudio; por tanto, se identifica el grado de influencia de cada una para considerarlo en el bosquejo de los posibles escenarios. Complementariamente, se menciona los métodos MACTOR (Postura de actores en un escenario futuro) y SMIC (Impactos cruzados probabilísticos).

En esta perspectiva se realzan los aportes de Godet (2000, 2007), fundador de LIPSOR (Lien Innovation, Prospective, Stratégie et Organisation) y quien, junto a su grupo de trabajo, desarrollo software para realizar estudios basados en el método francés de prospectiva. Los programas correspondientes son: MICMAC, MORPHOL, MULTIPOL, MACTOR y SMIC. El método
MACTOR se basa en el análisis de juego de actores buscando establecer las relaciones de fuerza existentes entre ellos. Por otra parte, con el SMIC se generan los escenarios posibles a partir de las apreciaciones que han tenido los expertos consultados sobre la realización de un grupo de hipótesis. Dentro de la clasificación de los escenarios se encuentra el tendencial (más probable), apuesta (deseado) y los cisnes negros (casos fortuitos emergentes).

Se destaca en la exposición de los autores el instructivo para el uso del software al describir minuciosamente cada una de las fases de implementación. Para este fin, los autores han colocado una reseña con los puntos neurálgicos de los programas, así como una secuencia de capturas de pantalla que facilitan la comprensión de las etapas implicadas. De esto derivan los ejemplos visuales que se procede a detallar:

\section{MACTOR}

- Ingreso de datos: Actores

- Ingreso de datos: Objetivos estratégicos

- Ingreso de datos: Matriz de influencias directas

- Ingreso de datos: Matriz de posiciones valoradas de actores sobre objetivos

- Menú de información de salida en MACTOR

- Resultados: Matriz $1 \mathrm{MAO}$

- Resultados: Matriz 2MAO

- Resultados: Matriz 3MAO

- Resultados: Gráfico de convergencias de orden 3

- Resultados: Gráfico de divergencias de orden 3 
- Resultados: Gráfico de convergencias de orden 1

- Resultados: Gráfico de divergencias de orden 1 SMIC

- Ingreso de datos: Hipótesis

- Ingreso de datos: Expertos y grupos de expertos

- Ingreso de datos: El mismo experto direccionando a un grupo de expertos

- Ingreso de datos: Lista de expertos

- Ingreso de datos: Selección de expertos en probabilidades simples

- Ingreso de datos: Probabilidades simples por experto

- Ingreso de datos: Probabilidades de si-si realización por experto

- Ingreso de datos: Probabilidades si-no realización por experto

- Menú de información de salida en SMIC

- Resultados: Matriz de soluciones contrastadas por grupo de expertos

- Resultados: Matriz de probabilidades de los escenarios. Grupo de expertos.

El modelamiento de macroentornos y de organizaciones individualizadas se aborda a partir de la dinamización acelerada en el aspecto tecnológico empresarial que, principalmente, genera una alta complejidad aunada a una elevada indeterminación, dando como resultando considerables niveles de incertidumbre. De esta manera, el bien transable preponderante es el conocimiento y este debe orientarse a la consecución de valor agregadoen productos y servicios con base en la innovación tecnológica.

De forma complementaria, también se expone el análisis prospectivo en espectros sociales $u$ organizacionales (modelamiento a futuro de macroentornos). Para tal efecto, se desarrolla un ejemplo completo que vincula la teoría y la práctica. Éste se centra en los servicios educativos en el distrito de San Juan de Lurigancho, Perú. Es necesario destacar la profundidad y la amplitud del caso presentado, puesto que contempla conceptos, uso de software, sustento matemático, análisis e interpretación de resultados. En otras palabras, abarca a plenitud el proceso de planeación estratégica prospectiva.

También se presenta al análisis prospectivo como plan de negocios de una empresa. Dicho de otro modo, se expone un modelamiento futuro de organizaciones individualizadas. Se presenta a manera de ejemplo un caso real, el cual consiste en la planeación de Industrias Plus Romantic E.I.R.L, ubicada en Lima. Este ejemplo, es ideal para comprender la apropiada de integración de los métodos de la escuela francesa y la planeación tradicional para entidades privadas, debido a que el nivel de especificación por cada fase es bastante alto, exhibiendo la forma en que se deben elaborar, aplicar e ingresar en SMIC los resultados de las encuestas tipo Delphi.

Como conclusión, al contrastar las indicaciones para el uso de los métodos MACTOR y SMIC con las experiencias personales previas en estudios prospectivos es posible aseverar que los autores se ajustan a la concepción de estudios de futuro plasmada por Godet (2000, 2007) en sus textos, principalmente con La Caja de Herramientas de la Prospectiva Estratégica y El Manual de Prospectiva Estratégica, así como el trabajo conjunto de Godet \& Durance (2011). En los ejemplos sociales y empresariales presentados, sacan el máximo provecho de las funcionalidades que tienen las herramientas y explican cómo ajustarlas para conseguir una abstracción más representativa de la realidad del caso de estudio. Sin embargo, aún es ausente una edición que incluya el encadenamiento y aplicación secuencial de la totalidad del software disponible, como se presenta en la figura 1.

Con base en lo expuesto, es notable que el libro ahonda en dos de los cinco métodos que componen el proceso básico de un estudio de esta naturaleza. En consecuencia, a pesar de describir trabajos de planificación íntegros como ejemplos, desaprovecha 
las potencialidades contenidas en MICMAC para clasificar y describir las relaciones entre variables, en MORPHOL con la capacidad para desarticular sistemas en elementos o componentes tipo Zwicky box y generar espacios morfológicos y, por último, en MULTIPOL para la estructuración de un tablero de análisis que pondere y priorice las acciones con el propósito de facilitar la toma de decisiones. A esto se podrían sumar herramientas con mayor nivel de detalle, tal como el Ábaco de Régnier, Color Insight y la rueda de futuros.

Fuente: Elaboración del autor

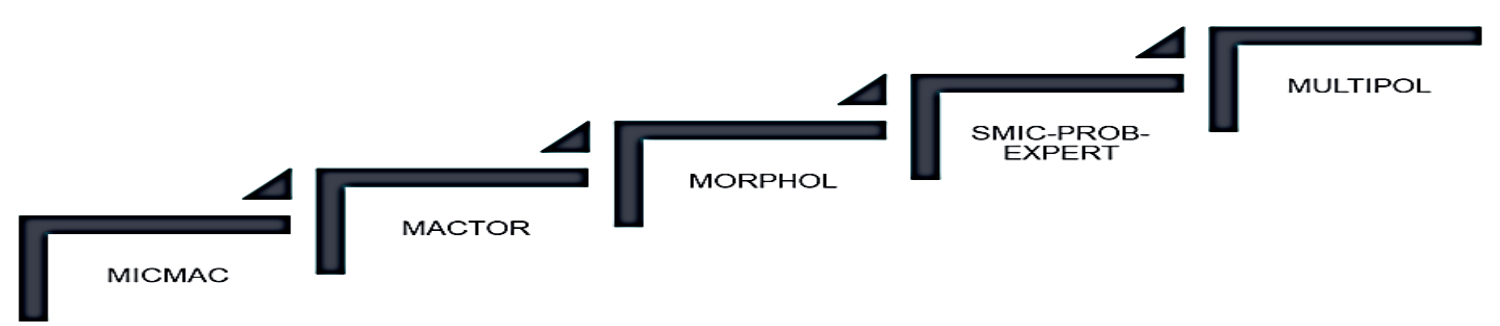

Figura 1. Fases básicas del estudio prospectivo acorde a la escuela francesa.

\section{REFERENCIAS}

Godet, Michel (2000). La Caja de Herramientas de la Prospectiva Estratégica (Cuarta ed.). París: Prospektiker. Godet, Michel (2007). El Manual de Prospectiva Estratégica. París: DUNOD.

Godet, Michel \& Durance, Philippe (2011). La Prospectiva Estratégica para las Empresas y los Territorios. París: DUNOD.

Norton, David \& Kaplan, Robert (1996). The Balanced ScoreCard: Translating Strategy into Action. Brighton: Harvard Business Press. Porter, Michael (2010). Ventaja Competitiva. Madrid: Pirámide.

Quinteros, José \& Hamann, Antonieta (2017). Planeamiento Estratégico Prospectivo: Métodos MACTOR y SMIC. Bogotá, ECOE Ediciones

\section{NOTAS FINALES DEL ARTÍCULO}

Reseña del libro de Quinteros \& Hamann (2017). Artículo adelantado en la Pontificia Universidad Católica de Ecuador, http://www.pucesd.edu.ec/, Santo Domingo de los Tsáchilas, Ecuador. Fecha de recepción 26/09/2019. Fecha de aceptación 30/11/2019.

ii Magister en Planificación y Prospectiva Multisectorial, docente en la Escuela de Ciencias Administrativas y Contables de la Pontificia Universidad Católica del Ecuador, sede Santo Domingo. Correo electrónico: cghernandezo@pucesd.edu.ec

Este artículo fue editado en la Facultad de Ciencias Administrativas, Económicas y Contables, www.uac.edu.co/facultades/facultad-ciencias-administrativas-economicas-y-contables, de la Universidad Autónoma del 